\title{
Publisher's Note: Engineering a Robust Quantum Spin Hall State in Graphene via Adatom Deposition [Phys. Rev. X 1, 021001 (2011)]
}

\section{Conan Weeks, Jun Hu, Jason Alicea, Marcel Franz, and Ruqian Wu (Received 28 March 2012; published 5 April 2012)}

DOI: $10.1103 /$ PhysRevX.2.029901

Subject Areas: Graphene, Condensed Matter Physics

This paper was published online on 3 October 2011 with an omission in the Acknowledgments. "J.H. and R. W. acknowledge support from the DOE-BES (Grant No. DE-FG02-05ER46237) and computing time on NERSC supercomputers." has been added to the Acknowledgments as of 30 March 2012. 